\title{
Are short GRBs powered by magnetars?
}

\author{
Paul T. O'Brien ${ }^{1}$ and Antonia Rowlinson ${ }^{2}$ \\ ${ }^{1}$ Department of Physics \& Astronomy, University of Leicester \\ University Road, Leicester, LE1 7RH, United Kingdom \\ email: paul.obrien@leicester.ac.uk \\ ${ }^{2}$ Astronomical Institute "Anton Pannekoek", University of Amsterdam, Postbus 94249, 1090 \\ GE Amsterdam, The Netherlands \\ email: b.a.rowlinson@uva.nl
}

\begin{abstract}
The standard model for a short duration Gamma-Ray Burst (GRB) involves the merger of a neutron star binary system, resulting in a black hole which accretes for a brief period of time. However, some of the short-duration GRBs observed by the Swift satellite show features in their light curves which are difficult to explain in this model. As an alternative, we examine the light curves of the Swift short GRB sample to see if they can be explained by the presence of a highly magnetised, rapidly rotating pulsar, or magnetar. We find that magnetars may be present in a large fraction of short bursts, and discuss briefly how this model can be tested using the next generation of gravity-wave observatories.
\end{abstract}

Keywords. gamma rays: bursts, (stars:) pulsars: general, black hole physics

\section{Introduction}

The Swift satellite (Gehrels et al. 2004) has observed a number of short gamma-ray bursts (SGRBs) X-ray afterglows (e.g. Gehrels et al. 2005). The properties of the light curves and their inferred host galaxies have provided support for the most popular compact binary merger progenitor theory, i.e. the coalescence of two neutron stars (e.g. Lattimer \& Schramm 1976). However, without the coincident observation of gravitational waves by observatories like LIGO (Laser Interferometry Gravitational-wave Observatory) we are missing the "smoking gun" observation for this progenitor theory. It is interesting that, as with the long-duration GRBs (LGRBs), many of the SGRBs show features in their X-ray light curves which suggest a long-lived central engine, for example late time flares and plateaus (e.g., Nousek et al. 2006; O'Brien et al. 2006).

The presence of long-lived features is particularly problematic for SGRB progenitor theories as accretion is expected to end within a few seconds and only a small fraction of the merger mass is available $\left(0.01-0.1 M_{\odot}\right)$, although this is dependant on the NS equation of state. An alternative energy source, if one can be found, is an attractive solution. One such source is that in some GRBs rather than a black hole (BH), a highly magnetised, rapidly rotating pulsar, or magnetar, may be formed with enough rotational energy to prevent gravitational collapse (e.g., Usov 1992; Duncan \& Thompson 1992). The rotational energy is then released as gravitational waves and electromagnetic radiation, causing the magnetar to spin down. If the magnetar is sufficiently massive it may reach a critical point at which differential rotation is no longer able to support it, resulting in collapse to a BH. Assuming constant radiative efficiency, the energy injection from the magnetar would produce a plateau in the X-ray light curve Zhang \& Mészáros (2001) and would be followed by a steep decay if the magnetar collapses to a $\mathrm{BH}$ or a more gentle decline if it does not collapse. 
We have recently proposed candidates for such a multi-stage progenitor system among both LGRBs (Troja et al. 2007; [Lyons et al. 2010]lyons2009) and SGRBs (Rowlinson et al. 2010). The likelihood of producing a magnetar is dependent on the equation of state of neutron stars. Morrison, Baumgarte, \& Shapiro (2004) showed that the rotation of the NS could increase the maximum mass by $\sim 50 \%$ and hence NS mergers could often result in a NS. Ozel et al. (2010) further showed that in such a merger the collapse to a $\mathrm{BH}$ can be delayed or not occur at all. Thus, it seems reasonable to assume that many NS binary mergers could result in a magnetar in a SGRB.

Here we consider all Swift detected SGRBs with $\mathrm{T}_{90} \leqslant 2$ s, observed until March 2012 with an X-ray afterglow or which were promptly slewed to and observed by the X-ray Telescope (XRT), and identify those with a plateau phase in their light-curves suggesting ongoing central engine activity. For the 28 SGRBs with sufficient data, we fit the 0.3$10 \mathrm{Kev}$ X-ray light curves to search for the signature of a magnetar (with or without collapse to a BH). This work is described in detail in Rowlinson et al. (2012, submitted to MNRAS).

\section{Magnetar model fits}

The model used here is as described in Zhang \& Mészáros (2001) and used by Troja et al. (2007), Lyons et al. (2010), Rowlinson et al. (2010). This model is consistent with the late-time residual spin-down phase driving a relativistic magnetar wind as described in Metzger et al. (2011). We fit the equations below with an additional underlying powerlaw component whose decay rate is governed by the curvature effect (Kumar \& Panaitescu 2000). We use the fitted values of the magnetic field and initial spin period to explain the luminosity of the X-ray plateau and its duration.

$$
\begin{array}{r}
B_{p, 15}^{2}=4.2025 I_{45}^{2} R_{6}^{-6} L_{0,49}^{-1} T_{e m, 3}^{-2} \\
P_{0,-3}^{2}=2.05 I_{45} L_{0,49}^{-1} T_{e m, 3}^{-1}
\end{array}
$$

where $T_{e m, 3}$ is the plateau duration in $10^{3} \mathrm{~s}, L_{0,49}$ is the initial plateau luminosity in $10^{49}$ erg s${ }^{-1}, I_{45}$ is the moment of inertia in units of $10^{45} \mathrm{~g} \mathrm{~cm}^{2}, B_{p, 15}$ is the magnetic field strength at the poles in units of $10^{15} \mathrm{G}, R_{6}$ is the radius of the neutron star in $10^{6} \mathrm{~cm}$ and $P_{0,-3}$ is the initial period of the compact object in milliseconds. These equations apply to the electromagnetic dominated spin down regime. We have assumed that the
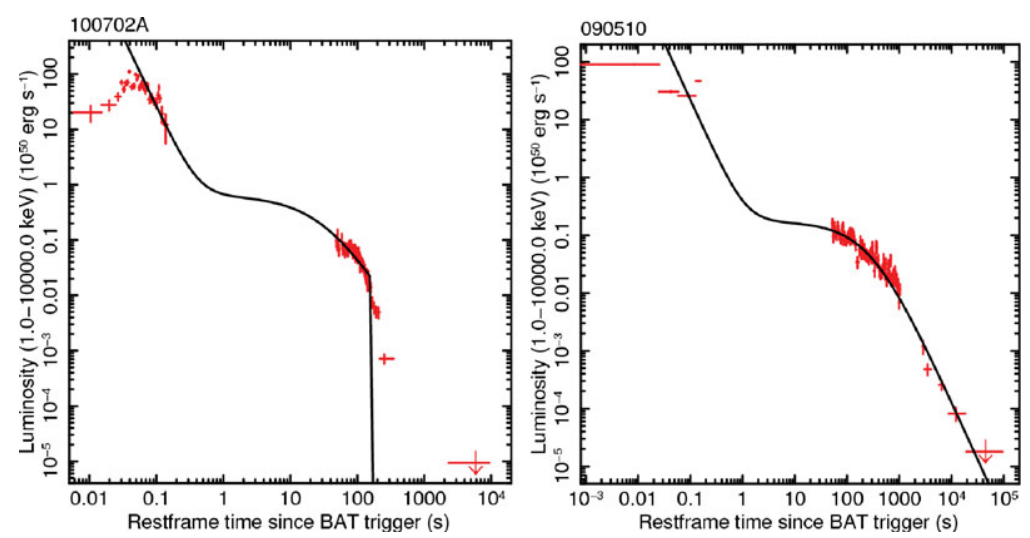

Figure 1. Model fit to two short GRBs: 100702A (left) best-fit by a magnetar which collapses to a black hole, and 090510 (right) which survives as a magnetar. 
emission is $100 \%$ efficient and isotropic. The equations of vacuum dipole spin-down given above neglect the enhanced angular momentum losses due to neutrino-driven mass loss, which are important at early times after the magnetar forms Metzger et al. (2011). Nevertheless, these expressions reasonably approximate the spin-down of very highly magnetised neutron stars of most relevance in this paper. Isotropic emission is also a reasonable assumption for relatively powerful magnetar winds, since (unlike following the collapse of a massive star) the magnetar outflow cannot be confined efficiently by the relatively small quantity of surrounding material expected following a binary merger.

\section{Results}

Of the 28 SGRBs fitted, 21 provide a good or possible fit to the magnetar model $(75 \%)$ while the other 8 provide poor fits, although in some cases this may simply be due to insufficient data. Example fits are shown in Fig. 1 for GRB 100702A and GRB090510. GRB 100702A shows a sharp drop which is consistent with collapse of the magnetar to a $\mathrm{BH}$ (after 167s in this case). For GRB 090510, the fit does not require collapse to a BH so we class that as an object where the magnetar survives. Among the 21 SGRBs with good/possible fits, 8 collapse to a $\mathrm{BH}(38 \%)$ within the first few hundred seconds.

The derived magnetic field strengths and initial spin periods are shown in Fig. 2 for the 28 SGRBs fitted. All objects are to the right of the shortest allowed spin period. All of the magnetar candidates are in the $10^{15} \mathrm{G} \leqslant B \leqslant 10^{17} \mathrm{G}$ region, but some are rotating relatively slowly, particularly where they do not require a collapse to a $\mathrm{BH}$.

\section{Gravitational wave signals}

If the magnetar model we propose here is correct, gravitational wave signals may be detectable from all three stages the systems can be in: inspiral to form magnetar, spin down and collapse to BH. In Table 1 we give a comparison of the distances to

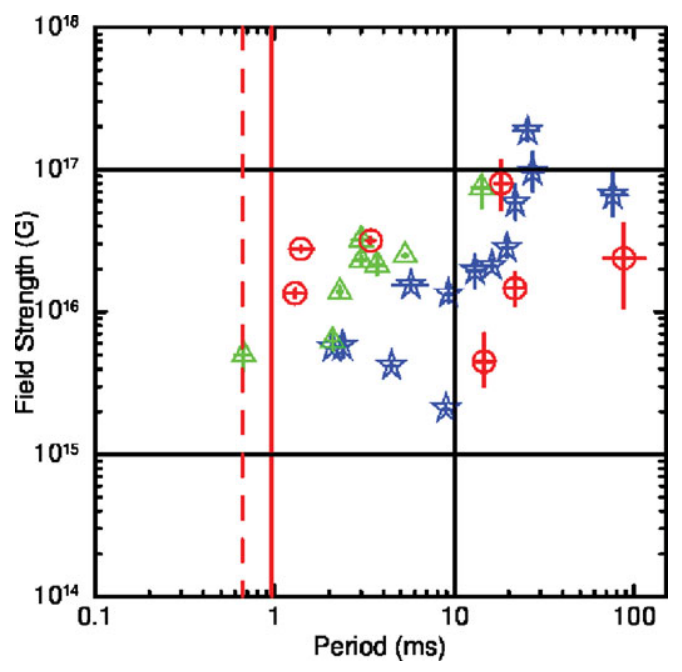

Figure 2. The derived magnetic fields and spin periods. The solid (dashed) vertical lines near $1 \mathrm{~ms}$ represent the spin break up periods for a $1.4 \mathrm{M}_{\odot}$ and $2.1 \mathrm{M}_{\odot}$ NS respectively (Lattimer \& Prakash 2004). The allowed region for an unstable magnetar is assumed such that the initial rotation period needs to be $\leqslant 10 \mathrm{~ms}$ (Usov 1992) and the magnetic field $10^{15} \mathrm{G} \leqslant B \leqslant 10^{17} \mathrm{G}$ (Thompson 2007). Stars: good fit with a stable magnetar; circles: good fit with an unstable magnetar which collapses to a $\mathrm{BH}$; and triangles: poor fit to the model. 


\begin{tabular}{lcc}
\hline Phase & $\begin{array}{c}\text { A-LIGO limit } \\
(\mathrm{Mpc})\end{array}$ & $\begin{array}{c}\text { ET Limit } \\
(\mathrm{Mpc})\end{array}$ \\
\hline Inspiral (Abadie et al. (2010)) & 445 & 5900 \\
Magnetar Spindown (Corsi \& Mészáros (2009)) & $<85$ & $<570$ \\
Collapse to BH (Novak (1998)) & 100 & 1300 \\
\hline
\end{tabular}

Table 1. Gravitational wave luminosity distance limits for A-LIGO and ET for the different regimes in this magnetar model (based on the predicted amplitudes given in the listed references).

which these signals could be detected by Advanced LIGO (A-LIGO) and the proposed Einstein Telescope (ET) based on the gravitational wave amplitudes discussed in the cited references and assuming a sensitivity of $h \sim 4 \times 10^{-24}$ for A-LIGO and $h \sim 3 \times 10^{-25}$ (Hild et al. 2011) for ET.

The chances of a simultaneous electromagnetic and gravitational wave detection are modest for A-LIGO but high for ET. The detection of such multiple gravitational wave signals corresponding to distinct light curve features would be a "smoking gun" test of the magnetar model.

\section{References}

Abadie J., et al., 2010, CQGra, 27, 173001

Corsi A. \& Mészáros P., 2009, ApJ, 702, 1171

Duncan R. C. \& Thompson C., 1992, ApJ, 392, L9

Gehrels N., et al., 2004, ApJ, 611, 1005

Gehrels, N., et al. 2005, Nature, 437, 851

Hild S., et al., 2011, CQGra, 28, 094013

Kumar P. \& Panaitescu A., 2000, ApJ, 541, L51

Lattimer, J. M. \& Schramm, D. N., 1976, ApJ, 210, 549

Lattimer J. M. \& Prakash M., 2004, Science, 304, 536

Lyons N., O'Brien P. T., Zhang B., Willingale R., Troja E., \& Starling R. L. C., 2010, MNRAS, 402, 705

Metzger B. D., Giannios D., Thompson T. A., Bucciantini N., \& Quataert E., 2011, MNRAS, 413, 2031

Morrison I. A., Baumgarte T. W., \& Shapiro S. L., 2004, ApJ, 610, 941

Nousek J. A., et al., 2006, ApJ, 642, 389

Novak J., 1998, Phys. Rev. D, 57, 4789

O'Brien, P. T., et al., 2006, ApJ, 647, 1213

Ozel F., Psaltis D., Ransom S., Demorest P., \& Alford M., 2010, ApJ, 724, L199

Rowlinson, A., et al. 2010, MNRAS, 409, 531

Thompson T. A., 2007, Rev. Mexicana AyA, 27, 80

Troja E., et al., 2007, ApJ, 665, 599

Usov V. V., 1992, Nature, 357, 472

Zhang B. \& Mészáros P., 2001, ApJ, 552, L35

\section{Discussion}

Corsi: In the unstable magnetar scenario, where is the afterglow emission and is there any spectral difference during the plateau phase between stable and unstable magnetar cases?

O'BRIEN: We include an underlying power-law component in the fits, which may be the afterglow or the off-axis (curvature) emission. Within the uncertainties, there are no spectral difference 